\title{
Emergency assessment of proximal left anterior descending coronary stent permeability using transthoracic echocardiography
}

\author{
Georgios Giannakopoulos@, Jose David Arroja, Alexandre Guinand, Hajo Müller \\ Cardiology Division, University Hospitals of Geneva, Switzerland
}

\begin{abstract}
A 44-year-old man with acute anterior ST elevation myocardial infarction underwent emergency percutaneous coronary intervention and stenting of an occluded ostial left anterior descending artery (LAD). Initial echocardiographic assessment showed anterior septal and apical akinesis. Thirty-six hours later, he developed a crushing chest pain that radiated to the back and the abdomen. Serial 12-lead electrocardiograms showed persistence of ST segment elevation that had not resolved since admission to the coronary unit. An emergency echocardiogram was performed. Using the left parasternal acoustic window, a short axis view at the level of the aortic valve was obtained and slightly modified by superiorly tilting the probe. Color Doppler flow mapping with the Nyquist limit lowered at $14 \mathrm{~cm} / \mathrm{s}$ allowed identification of the mid$\mathrm{LAD}$ and its bifurcation with a large septal branch
\end{abstract}

(Fig. 1A, Suppl. Video). Antegrade LAD flow was also demonstrated by the red color Doppler flow into the anterior interventricular groove (Fig. 1B) and using pulsed wave Doppler with the sampling volume placed inside the LAD (Fig. 1C). As the electrocardiogram was not contributing, these echocardiographic findings allowed the demonstration of at least partial permeability of the ostial LAD, excluding a complete occlusion of the stent, and provided time to obtain serial troponin samples. A decreasing troponin trend and further clinical evaluation-oriented investigations to the upper abdominal region and an acute cholecystitis was finally diagnosed by means of a thoraco-abdominal computed tomography scan.

Direct visualization of antegrade blood flow in the coronary arteries is feasible and can directly influence clinical decision making.

Conflict of interest: None declared

Address for correspondence: Georgios Giannakopoulos, MD, Hôpitaux Universitaires de Genève, Service de Cardiologie, Rue Gabrielle-Perret-Gentil 4, 1205 Genève, Switzerland, tel:+41795534469, e-mail: georgios.giannakopoulos@hcuge.ch Received: 14.04 .2020 Accepted: 2.07.2020

This article is available in open access under Creative Common Attribution-Non-Commercial-No Derivatives 4.0 International (CC BY-NC-ND 4.0) license, allowing to download articles and share them with others as long as they credit the authors and the publisher, but without permission to change them in any way or use them commercially. 


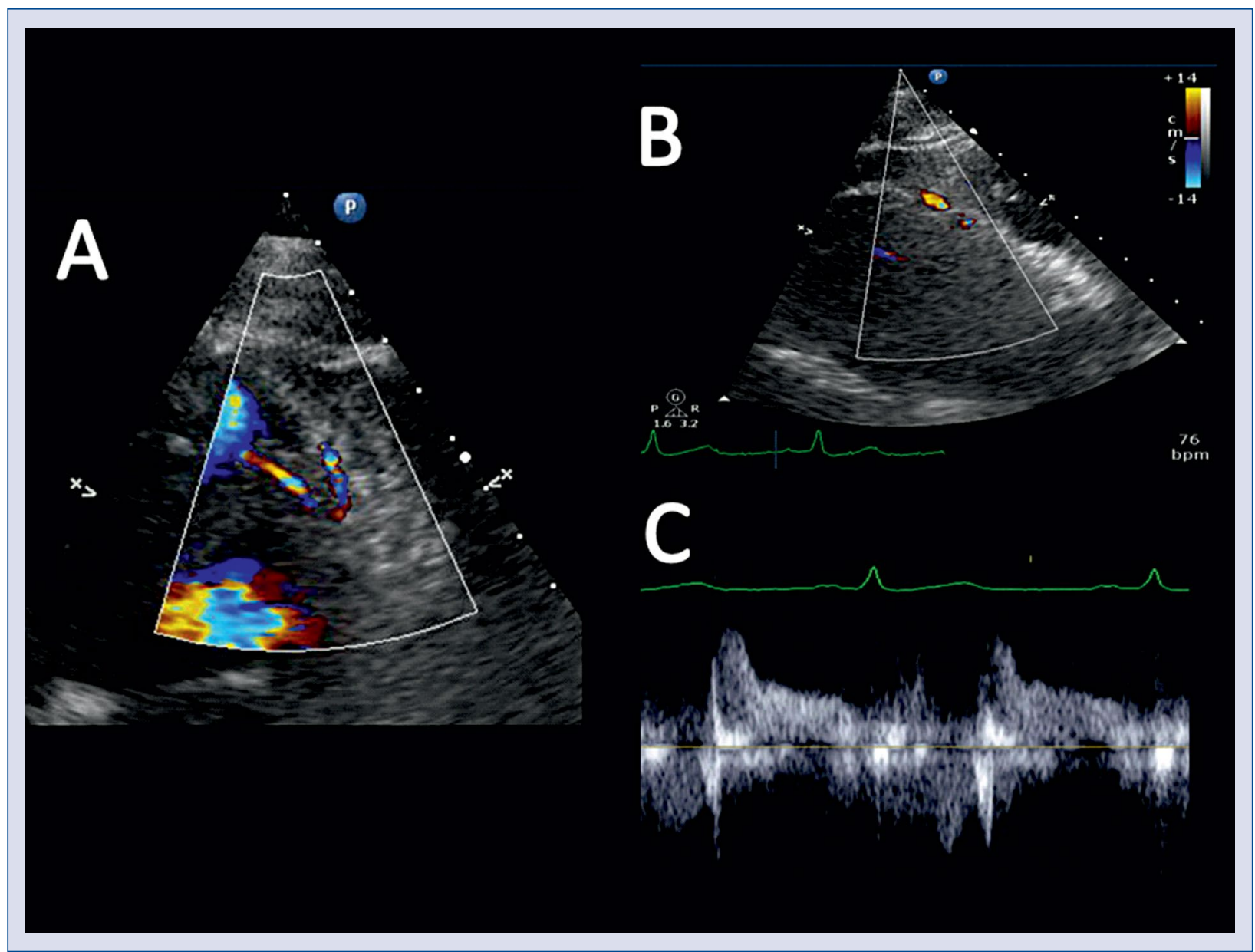

Figure 1. A. Modified parasternal short axis view. Color Doppler flow mapping in mid-diastole showing the bifurcation of left anterior descending artery (LAD) (to the right) with a large septal branch (to the left); B. Modified parasternal long axis view showing an antegrade (red) diastolic color Doppler flow in the anterior interventricular sulcus corresponding to the distal portion of the LAD; C. Pulsed wave Doppler with the sample volume positioned on the color Doppler signal of the LAD showing a predominately diastolic antegrade flow. 